\title{
Tagged Back-Translation
}

\author{
Isaac Caswell, Ciprian Chelba, David Grangier \\ Google Research \\ \{icaswell, ciprianchelba, grangier\}@google.com
}

\begin{abstract}
Recent work in Neural Machine Translation (NMT) has shown significant quality gains from noised-beam decoding during backtranslation, a method to generate synthetic parallel data. We show that the main role of such synthetic noise is not to diversify the source side, as previously suggested, but simply to indicate to the model that the given source is synthetic. We propose a simpler alternative to noising techniques, consisting of tagging back-translated source sentences with an extra token. Our results on WMT outperform noised back-translation in English-Romanian and match performance on English-German, re-defining state-of-the-art in the former.
\end{abstract}

\section{Introduction}

Neural Machine Translation (NMT) has made considerable progress in recent years (Bahdanau et al., 2015; Gehring et al., 2017; Vaswani et al., 2017). Traditional NMT has relied solely on parallel sentence pairs for training data, which can be an expensive and scarce resource. This motivates the use of monolingual data, usually more abundant (Lambert et al., 2011). Approaches using monolingual data for machine translation include language model fusion for both phrasebased (Brants et al., 2007; Koehn, 2009) and neural MT (Gülçehre et al., 2015, 2017), backtranslation (Sennrich et al., 2016; Poncelas et al., 2018), unsupervised machine translation (Lample et al., 2018a; Artetxe et al., 2018), dual learning (Cheng et al., 2016; Di He and Ma, 2016; Xia et al., 2017), and multi-task learning (Domhan and Hieber, 2017).

We focus on back-translation (BT), which, despite its simplicity, has thus far been the most effective technique (Sennrich et al., 2017; Ha et al., 2017; García-Martínez et al., 2017). Backtranslation entails training an intermediate target- to-source model on genuine bitext, and using this model to translate a large monolingual corpus from the target into the source language. This allows training a source-to-target model on a mixture of genuine parallel data and synthetic pairs from back-translation.

We build upon Edunov et al. (2018) and Imamura et al. (2018), who investigate BT at the scale of hundreds of millions of sentences. Their work studies different decoding/generation methods for back-translation: in addition to regular beam search, they consider sampling and adding noise to the one-best hypothesis produced by beam search. They show that sampled BT and noisedbeam BT significantly outperform standard BT, and attribute this success to increased source-side diversity (sections 5.2 and 4.4).

Our work investigates noised-beam BT (NoisedBT) and questions the role noise is playing. Rather than increasing source diversity, our work instead suggests that the performance gains come simply from signaling to the model that the source side is back-translated, allowing it to treat the synthetic parallel data differently than the natural bitext. We hypothesize that BT introduces both helpful signal (strong target-language signal and weak cross-lingual signal) and harmful signal (amplifying the biases of machine translation). Indicating to the model whether a given training sentence is back-translated should allow the model to separate the helpful and harmful signal.

To support this hypothesis, we first demonstrate that the permutation and word-dropping noise used by Edunov et al. (2018) do not improve or significantly degrade NMT accuracy, corroborating that noise might act as an indicator that the source is back-translated, without much loss in mutual information between the source and target. We then train models on WMT English-German (EnDe) without BT 
noise, and instead explicitly tag the synthetic data with a reserved token. We call this technique "Tagged Back-Translation" (TaggedBT). These models achieve equal to slightly higher performance than the noised variants. We repeat these experiments with WMT English-Romanian (EnRo), where NoisedBT underperforms standard BT and TaggedBT improves over both techniques. We demonstrate that TaggedBT also allows for effective iterative back-translation with EnRo, a technique which saw quality losses when applied with standard back-translation.

To further our understanding of TaggedBT, we investigate the biases encoded in models by comparing the entropy of their attention matrices, and look at the attention weight on the tag. We conclude by investigating the effects of the backtranslation tag at decoding time.

\section{Related Work}

This section describes prior work exploiting target-side monolingual data and discusses related work tagging NMT training data.

\subsection{Leveraging Monolingual Data for NMT}

Monolingual data can provide valuable information to improve translation quality. Various methods for using target-side LMs have proven effective for NMT (He et al., 2016; Gülçehre et al., 2017), but have tended to be less successful than back-translation - for example, Gülçehre et al. (2017) report under +0.5 BLEU over their baseline on EnDe newstest14, whereas Edunov et al. (2018) report over +4.0 BLEU on the same test set. Furthermore, there is no straighforward way to incorporate source-side monolingual data into a neural system with a LM.

Back-translation was originally introduced for phrase-based systems (Bertoldi and Federico, 2009; Bojar and Tamchyna, 2011), but flourished in NMT after work by Sennrich et al. (2016). Several approaches have looked into iterative forwardand BT experiments (using source-side monolingual data), including Cotterell and Kreutzer (2018), Vu Cong Duy Hoang and Cohn (2018), and Niu et al. (2018). Recently, iterative backtranslation in both directions has been devised has a way to address unsupervised machine translation (Lample et al., 2018b; Artetxe et al., 2018).

Recent work has focused on the importance of diversity and complexity in synthetic training data. Fadaee and Monz (2018) find that BT benefits difficult-to-translate words the most, and select from the back-translated corpus by oversampling words with high prediction loss. Imamura et al. (2018) argue that in order for BT to enhance the encoder, it must have a more diverse source side, and sample several back-translated source sentences for each monolingual target sentence. Our work follows most closely Edunov et al. (2018), who investigate alternative decoding schemes for BT. Like Imamura et al. (2018), they argue that BT through beam or greedy decoding leads to an overly regular domain on the source side, which poorly represents the diverse distribution of natural text.

Beyond the scope of this work, we briefly mention alternative techniques leveraging monolingual data, like forward translation (Ueffing et al., 2007; Kim and Rush, 2016), or source copying (Currey et al., 2017).

\subsection{Training Data Tagging for NMT}

Tags have been used for various purposes in NMT. Tags on the source sentence can indicate the target language in multi-lingual models (Johnson et al., 2016). Yamagishi et al. (2016) use tags in a similar fashion to control the formality of a translation from English to Japanese. Kuczmarski and Johnson (2018) use tags to control gender in translation. Most relevant to our work, Kobus et al. (2016) use tags to mark source sentence domain in a multi-domain setting.

\section{Experimental Setup}

This section presents our datasets, evaluation protocols and model architectures. It also describes our back-translation procedure, as well as noising and tagging strategies.

\subsection{Data}

We perform our experiments on WMT18 EnDe bitext, WMT16 EnRo bitext, and WMT15 EnFr bitext respectively. We use WMT Newscrawl for monolingual data (2007-2017 for De, 2016 for Ro, 2007-2013 for En, and 2007-2014 for Fr). For bitext, we filter out empty sentences and sentences longer than 250 subwords. We remove pairs whose whitespace-tokenized length ratio is greater than 2. This results in about 5.0M pairs for EnDe, and $0.6 \mathrm{M}$ pairs for EnRo. We do not filter the EnFr bitext, resulting in $41 \mathrm{M}$ sentence pairs. 
For monolingual data, we deduplicate and filter sentences with more than 70 tokens or 500 characters. Furthermore, after back-translation, we remove any sentence pairs where the back-translated source is longer than 75 tokens or 550 characters. This results in $216.5 \mathrm{M}$ sentences for EnDe, $2.2 \mathrm{M}$ for EnRo, 149.9M for RoEn, and 39M for EnFr. For monolingual data, all tokens are defined by whitespace tokenization, not wordpieces.

The DeEn model used to generate BT data has 28.6 SacreBLEU on newstest12, the RoEn model used for BT has a test SacreBLEU of 31.9 (see Table 4.b), and the FrEn model used to generate the BT data has 39.2 SacreBLEU on newstest 14 .

\subsection{Evaluation}

We rely on BLEU score (Papineni et al., 2002) as our evaluation metric.

While well established, any slight difference in post-processing and BLEU computation can have a dramatic impact on output values (Post, 2018). For example, Lample and Conneau (2019) report 33.3 BLEU on EnRo using unsupervised NMT, which at first seems comparable to our reported 33.4 SacreBLEU from iterative TaggedBT. However, when we use their preprocessing scripts and evaluation protocol, our system achieves 39.2 BLEU on the same data, which is close to 6 points higher than the same model evaluated by SacreBLEU.

We therefore report strictly SacreBLEU ${ }^{1}$, using the reference implementation from Post (2018), which aims to standardize BLEU evaluation.

\subsection{Architecture}

We use the transformer-base and transformer-big architectures (Vaswani et al., 2017) implemented in lingvo (Shen et al., 2019). Transformer-base is used for the bitext noising experiments and the EnRo experiments, whereas the transformer-big is used for the EnDe tasks with BT. Both use a vocabulary of $32 \mathrm{k}$ subword units. As an alternative to the checkpoint averaging used in Edunov et al. (2018), we train with exponentially weighted moving average (EMA) decay with weight decay parameter $\alpha=0.999$ (Buduma and Locascio, 2017).

Transformer-base models are trained on 16 GPUs with synchronous gradient updates and pergpu-batch-size of 4,096 tokens, for an effective

\footnotetext{
${ }^{1}$ BLEU + case.mixed + lang.LANGUAGE_PAIR + numrefs. $1+$ smooth.exp + test.SET + tok.13a + version.1.2.15
}

batch size of $64 \mathrm{k}$ tokens/step. Training lasts $400 \mathrm{k}$ steps, passing over 24B tokens. For the final EnDe TaggedBT model, we train transformer-big similarly but on 128 GPUs, for an effective batch size of $512 \mathrm{k}$ tokens/step. A training run of $300 \mathrm{M}$ steps therefore sees about 150B tokens. We pick checkpoints with newstest2012 for EnDe and newsdev2016 for EnRo.

\subsection{Noising}

We focused on noised beam BT, the most effective noising approach according to Edunov et al. (2018). Before training, we noised the decoded data (Lample et al., 2018a) by applying $10 \%$ worddropout, $10 \%$ word blanking, and a 3-constrained permutation (a permutation such that no token moves further than 3 tokens from its original position). We refer to data generated this way as NoisedBT. Additionally, we experiment using only the 3-constrained permutation and no word dropout/blanking, which we abbreviate as P3BT.

\subsection{Tagging}

We tag our BT training data by prepending a reserved token to the input sequence, which is then treated in the same way as any other token. We also experiment with both noising and tagging together, which we call Tagged Noised Back-Translation, or TaggedNoisedBT. This consists simply of prepending the $<\mathrm{BT}>$ tag to each noised training example.

An example training sentence for each of these set-ups can be seen in Table 1. We do not tag the bitext, and always train on a mix of backtranslated data and (untagged) bitext unless explicitly stated otherwise.

\begin{tabular}{l|l} 
Noise type & Example sentence \\
\hline [no noise] & $\begin{array}{l}\text { Raise the child, love the child. } \\
\text { child Raise the, love child the. }\end{array}$ \\
P3BT & Raise child love child, the. \\
NoisedBT & $<$ BT $>$ Raise the child, love the child. \\
TaggedBT & $<$ BT $>$ Raise, the child the love.
\end{tabular}

Table 1: Examples of the five noising settings examined in this paper

\section{Results}

This section studies the impact of training data noise on translation quality, and then presents our results with TaggedBT on EnDe and EnRo. 


\subsection{Noising Parallel Bitext}

We first show that noising EnDe bitext sources does not seriously impact the translation quality of the transformer-base baseline. For each sentence pair in the corpus, we flip a coin and noise the source sentence with probability $p$. We then train a model from scratch on this partially noised dataset. Table 2 shows results for various values of $p$. Specifically, it presents the somewhat unexpected finding that even when noising $100 \%$ of the source bitext (so the model has never seen wellformed English), BLEU on well-formed test data only drops by 2.5 .

This result prompts the following line of reasoning about the role of noise in BT: (i) By itself, noising does not add meaningful signal (or else it would improve performance); (ii) It also does not damage the signal much; (iii) In the context of back-translation, the noise could therefore signal whether a sentence were back-translated, without significantly degrading performance.

\begin{tabular}{l|cc} 
& \multicolumn{2}{|c}{ SacreBLEU } \\
$\%$ noised & Newstest'12 & Newstest'17 \\
\hline \hline $0 \%$ & 22.4 & 28.1 \\
$20 \%$ & 22.4 & 27.9 \\
$80 \%$ & 21.5 & 27.0 \\
$100 \%$ & 21.2 & 25.6
\end{tabular}

Table 2: SacreBLEU degradation as a function of the proportion of bitext data that is noised.

\subsection{Tagged Back-Translation for EnDe}

We compare the results of training on a mixture of bitext and a random sample of $24 \mathrm{M}$ backtranslated sentences in Table 3.a, for the various set-ups of BT described in sections 3.4 and 3.5. Like Edunov et al. (2018), we confirm that BT improves over bitext alone, and noised BT improves over standard BT by about the same margin. All methods of marking the source text as back-translated (NoisedBT, P3BT, TaggedBT, and TaggedNoisedBT) perform about equally, with TaggedBT having the highest average BLEU by a small margin. Tagging and noising together (TaggedNoisedBT) does not improve over either tagging or noising alone, supporting the conclusion that tagging and noising are not orthogonal signals but rather different means to the same end.

Table 3.b verifies our result at scale applying TaggedBT on the full BT dataset $(216.5 \mathrm{M}$ sentences), upsampling the bitext so that each batch contains an expected $20 \%$ of bitext. As in the smaller scenario, TaggedBT matches or slightly out-performs NoisedBT, with an advantage on seven test-sets and a disadvantage on one. We also compare our results to the best-performing model from Edunov et al. (2018). Our model is on par with or slightly superior to their result $^{2}$, out-performing it on four test sets and underperforming it on two, with the largest advantage on Newstest2018 (+1.4 BLEU).

As a supplementary experiment, we consider training only on BT data, with no bitext. We compare this to training only on NoisedBT data. If noising in fact increases the quality or diversity of the data, one would expect the NoisedBT data to yield higher performance than training on unaltered BT data, when in fact it has about 1 BLEU lower performance (Table 3.a, "BT alone" and "NoisedBT alone").

We also compare NoisedBT versus TaggedNoisedBT in a set-up where the bitext itself is noised. In this scenario, the noise can no longer be used by the model as an implicit tag to differentiate between bitext and synthetic BT data, so we expect the TaggedNoisedBT variant to perform better than NoisedBT by a similar margin to NoisedBT's improvement over BT in the unnoised-bitext setting. The last sub-section of Table 3. a confirms this.

\subsection{Tagged Back-Translation for EnRo}

We repeat these experiments for WMT EnRo (Table 4). This is a much lower-resource task than EnDe, and thus can benefit more from monolingual data. In this case, NoisedBT is actually harmful, lagging standard BT by -0.6 BLEU. TaggedBT closes this gap and passes standard BT by +0.4 BLEU, for a total gain of +1.0 BLEU over NoisedBT.

\subsection{Tagged Back-Translation for $\mathrm{EnFr}$}

We performed a minimal set of experiments on WMT EnFr, which are summarized in Table 5. This is a much higher-resource language pair than either EnRo or EnDe, but Edunov et al. (2018) demonstrate that noised BT (using sampling) can still help in this set-up. In this case, we see that BT alone hurts performance compared to the strong bitext baseline, but NoisedBT indeed surpasses the bitext model. TaggedBT out-performs all other

\footnotetext{
${ }^{2}$ SacreBLEU for the WMT-18 model at github.com/ pytorch/fairseq
} 


\begin{tabular}{|c|c|c|c|c|c|c|c|c|c|c|}
\hline \multicolumn{11}{|c|}{ a. Results on $24 \mathrm{M} \mathrm{BT}$ Set } \\
\hline Model & AVG 13-18 & 2010 & 2011 & 2012 & 2013 & 2014 & 2015 & 2016 & 2017 & 2018 \\
\hline$\overline{\text { Bitext }}$ & 32.05 & 24.8 & 22.6 & 23.2 & 26.8 & $\overline{28.5}$ & $\overline{\overline{31.1}}$ & $\overline{34.7}$ & $\overline{29.1}$ & 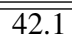 \\
\hline BT & 33.12 & 24.7 & 22.6 & 23.5 & 26.8 & 30.8 & 30.9 & 36.1 & 30.6 & 43.5 \\
\hline NoisedBT & 34.70 & 26.2 & 23.7 & 24.7 & 28.5 & 31.3 & 33.1 & 37.7 & 31.7 & 45.9 \\
\hline P3BT & 34.57 & 26.1 & 23.6 & 24.5 & 28.1 & 31.8 & 33.0 & 37.4 & 31.5 & 45.6 \\
\hline TaggedBT & 34.83 & 26.4 & 23.6 & 24.5 & 28.1 & 32.1 & 33.4 & 37.8 & 31.7 & 45.9 \\
\hline TaggedNoisedBT & 34.52 & 26.3 & 23.4 & 24.6 & 27.9 & 31.4 & 33.1 & 37.4 & 31.7 & 45.6 \\
\hline BT alone & 31.20 & 23.5 & 21.2 & 22.7 & 25.2 & 29.3 & 29.4 & 33.7 & 29.1 & 40.5 \\
\hline NoisedBT alone & 30.28 & 23.2 & 21.0 & 22.1 & 24.6 & 28.4 & 28.2 & 33.0 & 28.1 & 39.4 \\
\hline Noised(BT + Bitext) & 32.07 & 24.2 & 22.1 & 23.5 & 26.2 & 29.7 & 30.1 & 35.1 & 29.4 & 41.9 \\
\hline + Tag on BT & 33.53 & 25.5 & 22.8 & 24.5 & 27.6 & 30.3 & 31.9 & 36.9 & 30.4 & 44.1 \\
\hline \multicolumn{11}{|c|}{ b. Results on $216 \mathrm{M} \mathrm{BT} \mathrm{Set}$} \\
\hline Model & AVG $13-18$ & 2010 & 2011 & 2012 & 2013 & 2014 & 2015 & 2016 & 2017 & 2018 \\
\hline Edunov et al. (2018) & 35.28 & & & 25.0 & 29.0 & 33.8 & 34.4 & 37.5 & $\overline{32.4}$ & $\overline{444.6}$ \\
\hline NoisedBT & 35.17 & 26.7 & 24.0 & 25.2 & 28.6 & 32.6 & 33.9 & 38.0 & 32.2 & 45.7 \\
\hline TaggedBT & 35.42 & 26.5 & 24.2 & 25.2 & 28.7 & 32.8 & 34.5 & 38.1 & 32.4 & 46.0 \\
\hline
\end{tabular}

Table 3: SacreBLEU on Newstest EnDe for different types of noise, with back-translated data either sampled down to $24 \mathrm{M}$ or using the full set of $216 \mathrm{M}$ sentence pairs.

\begin{tabular}{l|cc}
\multicolumn{3}{c}{ a. Forward models (EnRo) } \\
Model & dev & test \\
\hline \hline Gehring et al. (2017) & \multicolumn{3}{c}{29.9} \\
Sennrich 2016 (BT) & 29.3 & 28.1 \\
\hline bitext & 26.5 & 28.3 \\
\hline BT & 31.6 & 32.6 \\
NoisedBT & 29.9 & 32.0 \\
TaggedBT & 30.5 & 33.0 \\
It.-3 BT & 31.3 & 32.8 \\
It.-3 NoisedBT & 31.2 & 32.6 \\
It.-3 TaggedBT & 31.4 & $\mathbf{3 3 . 4}$ \\
\multicolumn{3}{|c}{ b. Reverse models (RoEn) } \\
Model & dev & test \\
\hline \hline bitext & 32.9 & 31.9 \\
It.-2 BT & 39.5 & $\mathbf{3 7 . 3}$
\end{tabular}

Table 4: Comparing SacreBlEu scores for different flavors of BT for WMT16 EnRo. Previous works' scores are reported in italics as they use detok.multi-bleu instead of SacreBlEU, so are not guaranteed to be comparable. In this case, however, we do see identical BLEU on our systems when we score them with detok.multi-bleu, so we believe it to be a fair comparison.

methods, beating NoisedBT by an average of +0.3 BLEU over all test sets.

It is worth noting that our numbers are lower than those reported by Edunov et al. (2018) on the years they report $(36.1,43.8$, and 40.9 on 2013 , 2014 , and 2015 respectively). We did not investigate this result. We suspect that this is an error/inoptimlaity in our set-up, as we did not optimize these models, and ran only one experiment for each of the four set-ups. Alternately, sampling could outperform noising in the large-data regime.

\subsection{Iterative Tagged Back-Translation}

We further investigate the effects of TaggedBT by performing one round of iterative backtranslation (Cotterell and Kreutzer, 2018; Vu Cong Duy Hoang and Cohn, 2018; Niu et al., 2018), and find another difference between the different varieties of BT: NoisedBT and TaggedBT allow the model to bootstrap improvements from an improved reverse model, whereas standard BT does not. This is consistent with our argument that data tagging allows the model to extract information out of each data set more effectively.

For the purposes of this paper we call a model trained with standard back-translation an Iteration-1 BT model, where the backtranslations were generated by a model trained only on bitext. We inductively define the Iteration-k BT model as that model which is trained on BT data generated by an Iteration-(k-1) BT model, for $k>1$. Unless otherwise specified, any BT model mentioned in this paper is an Iteration-1 BT model.

We perform these experiments on the EnglishRomanian dataset, which is smaller and thus better suited for this computationally expensive process. We used the (Iteration-1) TaggedBT model to generate the RoEn back-translated training data. Using this we trained a superior RoEn model, mixing $80 \%$ BT data with $20 \%$ bitext. Using this Iteration-2 RoEn model, we generated new EnRo BT data, which we used to train the Iteration-3 EnRo models. SacreBleU scores for all these models are displayed in Table 4. 


\begin{tabular}{l|ccccccccc} 
Model & Avg & 2008 & 2009 & 2010 & 2011 & 2012 & 2013 & 2014 & 2015 \\
\hline \hline Bitext & 32.8 & 26.3 & 28.8 & 32.0 & 32.9 & 30.1 & 33.5 & 40.6 & 38.4 \\
BT & 29.2 & 22.2 & 27.3 & 28.8 & 29.3 & 27.9 & 30.7 & 32.6 & 34.8 \\
NoisedBT & 33.8 & 26.8 & 29.9 & 33.4 & $\mathbf{3 3 . 9}$ & $\mathbf{3 1 . 3}$ & 34.3 & 42.3 & 38.8 \\
TaggedBT & $\mathbf{3 4 . 1}$ & $\mathbf{2 7 . 0}$ & $\mathbf{3 0 . 0}$ & $\mathbf{3 3 . 6}$ & $\mathbf{3 3 . 9}$ & 31.2 & $\mathbf{3 4 . 4}$ & $\mathbf{4 2 . 7}$ & $\mathbf{3 9 . 8}$ \\
\hline
\end{tabular}

Table 5: Results on WMT15 EnFr, with bitext, BT, NoisedBT, and TaggedBT.

We find that the iteration-3 BT models improve over their Iteration-1 counterparts only for NoisedBT $(+1.0 \mathrm{BLEU}$, dev+test avg) and TaggedBT $(+0.7$ BLEU, dev+test avg), whereas the Iteration-3 BT model shows no improvement over its Iteration-1 counterpart (-0.1 BLEU, dev+test avg). In other words, both techniques that (explicitly or implicitly) tag synthetic data benefit from iterative BT. We speculate that this separation of the synthetic and natural domains allows the model to bootstrap more effectively from the increasing quality of the back-translated data while not being damaged by its quality issues, whereas the simple BT model cannot make this distinction, and is equally "confused" by the biases in higher or lower-quality BT data.

An identical experiment with EnDe did not see either gains or losses in BLEU from iteration3 TaggedBT. This is likely because there is less room to bootstrap with the larger-capacity model. This said, we do not wish to read too deeply into these results, as the effect size is not large, and neither is the number of experiments. A more thorough suite of experiments is warranted before any strong conclusions can be made on the implications of tagging on iterative BT.

\section{Analysis}

In an attempt to gain further insight into TaggedBT as it compares with standard BT or NoisedBT, we examine attention matrices in the presence of the back translation tag and measure the impact of the tag at decoding time.

\subsection{Attention Entropy and Sink-Ratio}

To understand how the model treats the tag and what biases it learns from the data, we investigate the entropy of the attention probability distribution, as well as the attention captured by the tag.

We examine decoder attention (at the top layer) on the first source token. We define Attention Sink Ratio for index $j\left(\mathrm{ASR}_{j}\right)$ as the averaged attention over the $j$ th token, normalized by uniform atten- tion, i.e.

$$
\operatorname{ASR}_{j}(x, \hat{y})=\frac{1}{|\hat{y}|} \sum_{i=1}^{|\hat{y}|} \frac{\alpha_{i j}}{\tilde{\alpha}}
$$

where $\alpha_{i j}$ is the attention value for target token $i$ in hypothesis $\hat{y}$ over source token $j$ and $\tilde{\alpha}=\frac{1}{|x|}$ corresponds to uniform attention. We examine ASR on text that has been noised and/or tagged (depending on the model), to understand how BT sentences are treated during training. For the tagged variants, there is heavy attention on the tag when it is present (Table 6), indicating that the model relies on the information signalled by the tag.

Our second analysis probes word-for-word translation bias through the average source-token entropy of the attention probability model when decoding natural text. Table 6 reports the average length-normalized Shannon entropy:

$$
\tilde{\mathrm{H}}(x, \hat{y})=-\frac{1}{|\hat{y}|} \sum_{i=1}^{|\hat{y}|} \frac{1}{\log |x|} \sum_{j=1}^{|x|} \alpha_{i j} \log \left(\alpha_{i j}\right)
$$

The entropy of the attention probabilities from the model trained on BT data is the clear outlier. This low entropy corresponds to a concentrated attention matrix, which we observed to be concentrated on the diagonal (See Figure 1a and 1d). This could indicate the presence of word-by-word translation, a consequence of the harmful part of the signal from back-translated data. The entropy on parallel data from the NoisedBT model is much higher, corresponding to more diffuse attention, which we see in Figure $1 \mathrm{~b}$ and 1e. In other words, the wordfor-word translation biases in BT data, that were incorporated into the BT model, have been manually undone by the noise, so the model's understanding of how to decode parallel text is not corrupted. We see that TaggedBT leads to a similarly high entropy, indicating the model has learnt this without needing to manually "break" the literaltranslation bias. As a sanity check, we see that the entropy of the P3BT model's attention is also high, but is lower than that of the NoisedBT model, because P3 noise is less destructive. The one sur- 


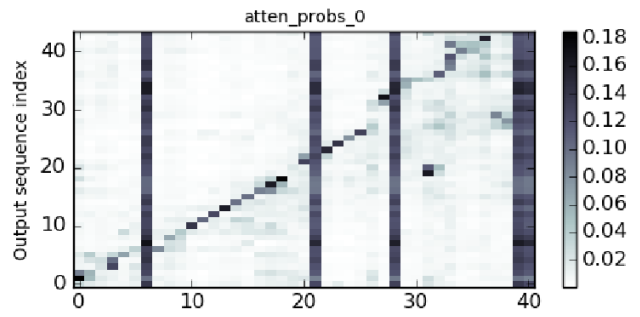

(a) EnDe BT

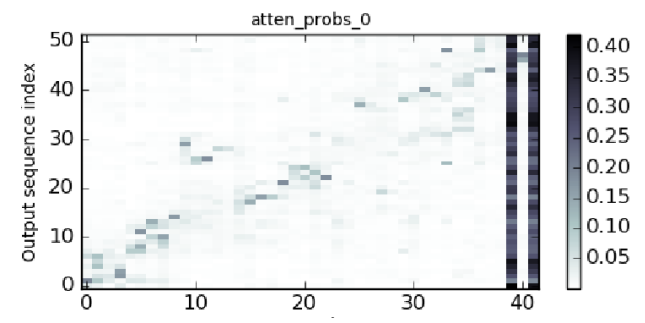

(b) EnDe NoisedBT

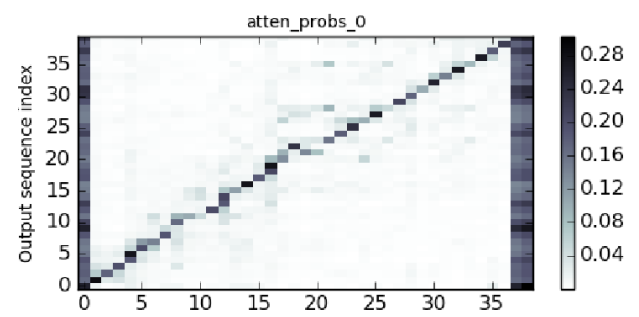

(c) EnDe TaggedBT

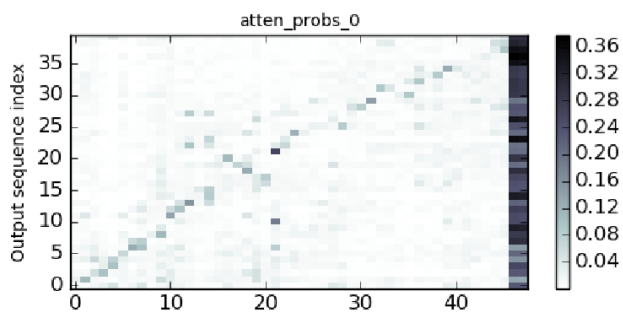

(d) EnRo BT

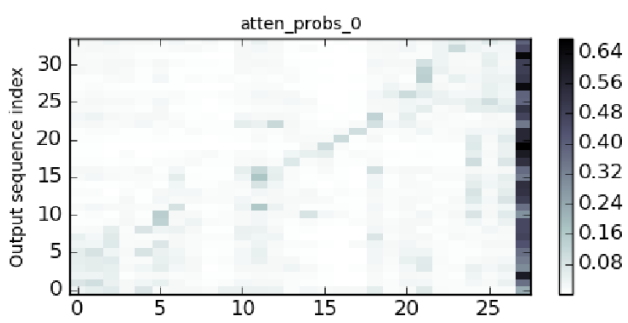

(e) EnRo NoisedBT

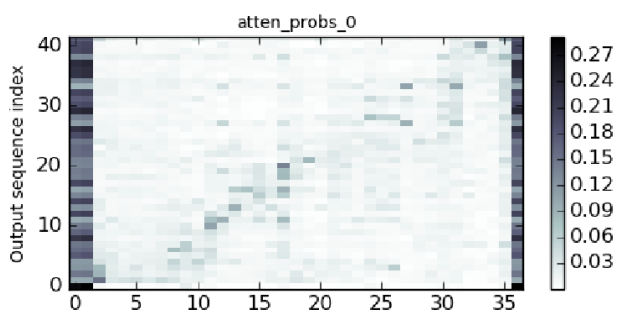

(f) EnRo TaggedBT

Figure 1: Comparison of attention maps at the first encoder layer for a random training example for BT (row 1), NoisedBT (row 2), and TaggedBT (row 3), for both EnDe (col 1) and EnRo (col 2). Note the heavy attention on the tag (position 0 in row 3 ), and the diffuse attention map learned by the NoiseBT models. These are the models from Table 3.a

prising entry on this table is probably the low entropy of the TaggedNoisedBT. Our best explanation is that TaggedNoisedBT puts disproportionately high attention on the sentence-end token, with 1.4x the $\mathrm{ASR}_{|x|}$ that TaggedBT has, naturally leading to lower entropy.

\begin{tabular}{l|c|c|c} 
Model & $\mathrm{ASR}_{0}$ & $\mathrm{ASR}_{|x|}$ & $\tilde{\mathrm{H}}$ \\
\hline \hline Bitext baseline & 0.31 & 10.21 & 0.504 \\
BT & 0.28 & 10.98 & $\mathbf{0 . 4 5 5}$ \\
P3BT & 0.37 & 7.66 & 0.558 \\
NoisedBT & 1.01 & 3.96 & 0.619 \\
TaggedBT & $\mathbf{5 . 3 1}$ & 5.31 & 0.597 \\
TaggedNoisedBT & $\mathbf{7 . 3 3}$ & 7.33 & 0.491
\end{tabular}

Table 6: Attention sink ratio on the first and last token and entropy (at decoder layer 5) for the models in Table 3.a, averaged over all sentences in newstest14. For ASR, data is treated as if it were BT (noised and/or tagged, resp.), whereas for entropy the natural text is used. Outliers discussed in the text are bolded.

\subsection{Decoding with and without a tag}

In this section we look at what happens when we decode with a model on newstest data as if it were back-translated. This means that for the TaggedBT model we tag the true source, and for the NoisedBT model, we noise the true source. These "as-if-BT" decodings contrast with "standard decode", or decoding with the true source. An example sentence from newstest 2015 is shown in Table 8 , decoded by both models both in the standard fashion and in the "as-if-BT" fashion. The BLEU scores of each decoding method are presented in Table 7.

The noised decode - decoding newstest sentences with the NoisedBT model after noising the source - yields poor performance. This is unsurprising given the severity of the noise model used (recall Table 1). The tagged decode, however, yields only somewhat lower performance than the standard decode on the same model (-2.9BLEU on average). There are no clear reasons for this qual- 


\begin{tabular}{l|lccccccccc} 
Model & Decode type & AVG 13-17 & 2010 & 2011 & 2012 & 2013 & 2014 & 2015 & 2016 & 2017 \\
\hline \hline TaggedBT & standard & $\mathbf{3 3 . 2 4}$ & 26.5 & $\mathbf{2 4 . 2}$ & $\mathbf{2 5 . 2}$ & $\mathbf{2 8 . 7}$ & $\mathbf{3 2 . 8}$ & $\mathbf{3 4 . 5}$ & $\mathbf{3 8 . 1}$ & $\mathbf{3 2 . 4}$ \\
& as BT (tagged) & 30.30 & 24.3 & 22.2 & 23.4 & 26.6 & 30.0 & 30.5 & 34.2 & 30.2 \\
\multirow{2}{*}{ NoisedBT } & standard & 33.06 & $\mathbf{2 6 . 7}$ & 24.0 & $\mathbf{2 5 . 2}$ & 28.6 & 32.6 & 33.9 & 38.0 & 32.2 \\
& as BT (noised) & 10.66 & 8.1 & 6.5 & 7.5 & 8.2 & 11.1 & 10.0 & 12.7 & 11.3 \\
\hline
\end{tabular}

Table 7: Comparing standard decoding with decoding as if the input were back-translated data, meaning that it is tagged (for the TaggedBT model) or noised (for the NoisedBT model) .

\begin{tabular}{|c|c|c|}
\hline Model & Decode type & Example \\
\hline TaggedBT & $\begin{array}{l}\text { standard } \\
\text { as-if-BT (tagged) } \\
\text { standard } \\
\text { as-if-BT (noised) }\end{array}$ & $\begin{array}{l}\text { Wie der Reverend Martin Luther King Jr. vor fünfzig Jahren sagte: } \\
\text { Wie sagte der Reverend Martin Luther King jr. Vor fünfzig Jahren: } \\
\text { Wie der Reverend Martin Luther King Jr. vor fünfzig Jahren sagte: } \\
\text { Als Luther King Reverend_ Jr. vor fünfzig Jahren: }\end{array}$ \\
\hline $\begin{array}{l}\text { Source } \\
\text { Reference }\end{array}$ & & $\begin{array}{l}\text { As the Reverend Martin Luther King Jr. said fifty years ago: } \\
\text { Wie Pastor Martin Luther King Jr. vor fünfzig Jahren sagte: }\end{array}$ \\
\hline
\end{tabular}

Table 8: Example decodes from newstest 2015 for decoding in standard and "as-if-BT" varieties. Here, NoisedBT and TaggedBT produce equivalent outputs with standard decoding; TaggedBT produces less natural output with tagged input; and NoisedBT produces a low-quality output with noised input.

ity drop - the model correctly omits the tag in the outputs, but simply produces slightly lower quality hypotheses. The only noticeable difference in decoding outputs between the two systems is that the tagged decoding produces about double the quantity of English outputs ( $2.7 \%$ vs. $1.2 \%$, over newstest2010-newstest2017, using a language ID classifier).

That the tagged-decode BLEU is still quite reasonable tells us that the model has not simply learned to ignore the source sentence when it encounters the input tag, suggesting that the $p(y \mid \mathrm{BT}(x))$ signal is still useful to the model, as Sennrich et al. (2016) also demonstrated. The tag might then be functioning as a domain tag, causing the model to emulate the domain of the BT data including both the desirable target-side news domain and the MT biases inherent in BT data.

To poke at the intuition that the quality drop comes in part from emulating the NMT biases in the synthetic training data, we probe a particular shortcoming of NMT: copy rate. We quantify the copy rate with the unigram overlap between source and target as a percentage of tokens in the target side, and compare those statistics to the bitext and the back-translated data (Table 9). We notice that the increase in unigram overlap with the tagged decode corresponds to the increased copy rate for the back-translated data (reaching the same value of $11 \%$ ), supporting the hypothesis that the tag helps the model separate the domain of the parallel versus the back-translated data. Under this lens, quality gains from TaggedBT/NoisedBT could be re-framed as transfer learning from a multi-task set-up, where one task is to translate simpler "translationese" (Gellerstam, 1986; Freitag et al., 2019) source text, and the other is to translate true bitext.

\begin{tabular}{l|c} 
Data & src-tgt unigram overlap \\
\hline \hline TaggedBT (standard decode) & $8.9 \%$ \\
TaggedBT (tagged decode) & $10.7 \%$ \\
Bitext & $5.9 \%$ \\
BT Data & $11.4 \%$ \\
\hline
\end{tabular}

Table 9: Source-target overlap for both back-translated data with decoding newstest as if it were bitext or BT data. Model decodes are averaged over newstest2010newstest2017.

\section{Negative Results}

In addition to tagged back-translation, we tried several tagging-related experiments that did not work as well. We experimented with tagged forward-translation (TaggedFT), and found that the tag made no substantial difference, often lagging behind untagged forward-translation (FT) by a small margin $(\sim 0.2 \mathrm{BLEU})$. For EnDe, (Tagged)FT underperformed the bitext baseline; for EnRo, (Tagged)FT performed about the same as BT. Combining BT and FT had additive effects, yielding results slightly higher than iteration-3 TaggedBT (Table 4), at 33.9 SacreBLEU on test; but tagging did not help in this set-up. We furthermore experimented with year-specific tags on the BT data, using a different tag for each of the ten years of newscrawl. The model trained on these data performed identically to the normal TaggedBT model. Using this model we repli- 
cated the "as-if-bt" experiments from Table 8 using year-specific tags, and although there was a slight correlation between year tag and that year's dataset, the standard-decode still resulted in the highest BLEU.

\section{Conclusion}

In this work we develop TaggedBT, a novel technique for using back-translation in the context of NMT, which improves over the current state-ofthe-art method of Noised Back-Translation, while also being simpler and more robust. We demonstrate that while Noised Back-Translation and standard Back-Translation are more or less effective depending on the task (low-resource, midresource, iterative BT), TaggedBT performs well on all tasks.

On WMT16 EnRo, TaggedBT improves on vanilla BT by 0.4 BLEU. Our best BLEU score of 33.4 BLEU, obtained using Iterative TaggedBT, shows a gain of +3.5 BLEU over the highest previously published result on this test-set that we are aware of. We furthermore match or out-perform the highest published results we are aware of on WMT EnDe that use only back-translation, with higher or equal BLEU on five of seven test sets.

In addition, we conclude that noising in the context of back-translation acts merely as an indicator to the model that the source is back-translated, allowing the model to treat it as a different domain and separate the helpful signal from the harmful signal. We support this hypothesis with experimental results showing that heuristic noising techniques like those discussed here, although they produce text that may seem like a nigh unintelligible mangling to humans, have a relatively small impact on the cross-lingual signal. Our analysis of attention and tagged decoding provides further supporting evidence for these conclusions.

\section{Future Work}

A natural extension of this work is to investigate a more fine-grained application of tags to both natural and synthetic data, for both backtranslation and forward-translation, using quality and domain tags as well as synth-data tags. Similarly, tagging could be investigated as an alternative to data selection, as in van der Wees et al. (2017); Axelrod et al. (2011), or curriculum learning approaches like fine-tuning on in-domain data (Thompson et al., 2018; Hassan Sajjad and Nadir
Durrani and Fahim Dalvi and Yonatan Belinkov and Stephan Vogel, 2017; Freitag and Al-Onaizan, 2016). Finally, the token-tagging method should be contrasted with more sophisticated versions of tagging, like concatenating a trainable domain embedding with all token embeddings, as in Kobus et al. (2016).

\section{Acknowledgements}

Thank you to Markus Freitag, Melvin Johnson and Wei Wang for advising and discussions about these ideas; thank you to Keith Stevens, Mia Chen, and Wei Wang for technical help and bug fixing; thank you to Sergey Edunov for a fast and thorough answer to our question about his paper; and of course to the various people who have given comments and suggestions throughout the process, including Bowen Liang, Naveen Arivazhagan, Macduff Hughes, and George Foster.

\section{References}

Mikel Artetxe, Gorka Labaka, and Eneko Agirre. 2018. Unsupervised Statistical Machine Translation. In Proceedings of the 2018 Conference on Empirical Methods in Natural Language Processing, pages 3632-3642.

Amittai Axelrod, Xiaodong He, and Jianfeng Gao. 2011. Domain adaptation via pseudo in-domain data selection. In Proceedings of the 2011 Conference on Empirical Methods in Natural Language Processing, pages 355-362.

Dzmitry Bahdanau, Kyunghyun Cho, and Yoshua Bengio. 2015. Neural Machine Translation by Jointly Learning to Align and Translate. In 3rd International Conference on Learning Representations, ICLR 2015.

Nicola Bertoldi and Marcello Federico. 2009. Domain adaptation for statistical machine translation with monolingual resources. In Proceedings of the fourth workshop on statistical machine translation, pages 182-189. Association for Computational Linguistics.

Ondřej Bojar and Aleš Tamchyna. 2011. Improving translation model by monolingual data. In Proceedings of the Sixth Workshop on Statistical Machine Translation, pages 330-336. Association for Computational Linguistics.

Thorsten Brants, Ashok C Popat, Peng Xu, Franz J Och, and Jeffrey Dean. 2007. Large Language Models in Machine Translation. In Proceedings of the 2007 Joint Conference on Empirical Methods in Natural Language Processing and Computational Natural Language Learning (EMNLP-CoNLL). 
Nikhil Buduma and Nicholas Locascio. 2017. Fundamentals of deep learning: Designing next-generation machine intelligence algorithms. "O’Reilly Media, Inc.".

Yong Cheng, Wei Xu, Zhongjun He, Wei He, Hua $\mathrm{Wu}$, Maosong Sun, and Yang Liu. 2016. SemiSupervised Learning for Neural Machine Translation. In Proceedings of the 54th Annual Meeting of the Association for Computational Linguistics, volume 1, pages 1965-1974.

Ryan Cotterell and Julia Kreutzer. 2018. Explaining and Generalizing Back-Translation through Wakesleep. arXiv preprint arXiv:1806.04402.

Anna Currey, Antonio Valerio Miceli Barone, and Kenneth Heafield. 2017. Copied monolingual data improves low-resource neural machine translation. In Proceedings of the Second Conference on Machine Translation, pages 148-156.

Tao Qin Liwei Wang Nenghai Yu Tieyan Liu Di He, Yingce Xia and Wei-Ying Ma. 2016. Dual Learning for Machine Translation. In Conference on Advances in Neural Information Processing Systems (NeurIPS).

Tobias Domhan and Felix Hieber. 2017. Using Targetside Monolingual Data for Neural Machine Translation through Multi-task Learning. In Proceedings of the 2017 Conference on Empirical Methods in Natural Language Processing, pages 1500-1505.

Sergey Edunov, Myle Ott, Michael Auli, and David Grangier. 2018. Understanding Back-Translation at Scale. In Proceedings of the 2018 Conference on Empirical Methods in Natural Language Processing (EMNLP), pages 489-500.

Marzieh Fadaee and Christof Monz. 2018. BackTranslation Sampling by Targeting Difficult Words in Neural Machine Translation. CoRR, abs/1808.09006.

Markus Freitag and Yaser Al-Onaizan. 2016. Fast Domain Adaptation for Neural Machine Translation. CoRR, abs/1612.06897.

Markus Freitag, Isaac Caswell, and Scott Roy. 2019. Text Repair Model for Neural Machine Translation. CoRR, abs/1904.04790.

Mercedes García-Martínez, Özan Çağlayan, Walid Aransa, Adrien Bardet, Fethi Bougares, and Loïc Barrault. 2017. LIUM Machine Translation Systems for WMT17 News Translation Task. CoRR, abs/1707.04499.

Jonas Gehring, Michael Auli, David Grangier, Denis Yarats, and Yann N. Dauphin. 2017. Convolutional Sequence to Sequence Learning. In Proceedings of the 34th International Conference on Machine Learning - Volume 70, pages 1243-1252.
Martin Gellerstam. 1986. Translationese in Swedish novels translated from English. Translation Studies in Scandinavia, pages 88-95.

Çağlar Gülçehre, Orhan Firat, Kelvin Xu, Kyunghyun Cho, Loic Barrault, Huei-Chi Lin, Fethi Bougares, Holger Schwenk, and Yoshua Bengio. 2015. On using Monolingual Corpora in Neural Machine Translation. arXiv preprint arXiv:1503.03535.

Çağlar Gülçehre, Orhan Firat, Kelvin Xu, Kyunghyun Cho, and Yoshua Bengio. 2017. On Integrating a Language Model into Neural Machine Translation. Comput. Speech Lang., pages 137-148.

Thanh-Le Ha, Jan Niehues, and Alexander H. Waibel. 2017. Effective Strategies in Zero-Shot Neural Machine Translation. CoRR, abs/1711.07893.

Hassan Sajjad and Nadir Durrani and Fahim Dalvi and Yonatan Belinkov and Stephan Vogel. 2017. Neural machine translation training in a multi-domain scenario. arXiv preprint arXiv:1708.08712v2.

Wei He, Zhongjun He, Hua Wu, and Haifeng Wang. 2016. Improved neural machine translation with SMT features. In Thirtieth AAAI conference on artificial intelligence.

Kenji Imamura, Atsushi Fujita, and Eiichiro Sumita. 2018. Enhancement of Encoder and Attention Using Target Monolingual Corpora in Neural Machine Translation. In Proceedings of the 2nd Workshop on Neural Machine Translation and Generation, volume 1, pages 55-63.

Melvin Johnson, Mike Schuster, Quoc V. Le, Maxim Krikun, Yonghui Wu, Zhifeng Chen, Nikhil Thorat, Fernanda B. Vi'egas, Martin Wattenberg, Greg Corrado, Macduff Hughes, and Jeffrey Dean. 2016. Google's Multilingual Neural Machine Translation System: Enabling Zero-Shot Translation. CoRR, abs/1611.04558.

Yoon Kim and Alexander M. Rush. 2016. SequenceLevel Knowledge Distillation. In Proceedings of the 2016 Conference on Empirical Methods in Natural Language Processing, pages 1317-1327, Austin, Texas. Association for Computational Linguistics.

Catherine Kobus, Josep Maria Crego, and Jean Senellart. 2016. Domain Control for Neural Machine Translation. CoRR, abs/1612.06140.

Philipp Koehn. 2009. Statistical machine translation. Cambridge University Press.

James Kuczmarski and Melvin Johnson. 2018. Gender-aware natural language translation. Technical Disclosure Commons.

Patrik Lambert, Holger Schwenk, Christophe Servan, and Sadaf Abdul-Rauf. 2011. Investigations on translation model adaptation using monolingual data. In Proceedings of the Sixth Workshop on Statistical Machine Translation, pages 284-293. Association for Computational Linguistics. 
Guillaume Lample and Alexis Conneau. 2019. Crosslingual Language Model Pretraining. arXiv preprint arXiv:1901.07291.

Guillaume Lample, Alexis Conneau, Ludovic Denoyer, and Marc'Aurelio Ranzato. 2018a. Unsupervised Machine Translation Using Monolingual Corpora Only. In International Conference on Learning Representations.

Guillaume Lample, Myle Ott, Alexis Conneau, Ludovic Denoyer, and Marc'Aurelio Ranzato. 2018b. Phrase-Based \& Neural Unsupervised Machine Translation. In Proceedings of the 2018 Conference on Empirical Methods in Natural Language Processing (EMNLP).

Xing Niu, Michael Denkowski, and Marine Carpuat. 2018. Bi-Directional Neural Machine Translation with Synthetic Parallel Data. ACL 2018, page 84.

Kishore Papineni, Salim Roukos, Todd Ward, and WeiJing Zhu. 2002. BLEU: a Method for Automatic Evaluation of Machine Translation. In Proceedings of the 40th annual meeting on association for computational linguistics, pages 311-318. Association for Computational Linguistics.

Alberto Poncelas, Dimitar Shterionov, Andy Way, Gideon Maillette de Buy Wenniger, and Peyman Passban. 2018. Investigating Backtranslation in Neural Machine Translation. In Proceedings of the 21st Annual Conference of the European Association for Machine Translation, pages 249-258.

Matt Post. 2018. A Call for Clarity in Reporting Bleu Scores. arXiv preprint arXiv:1804.08771.

Rico Sennrich, Alexandra Birch, Anna Currey, Ulrich Germann, Barry Haddow, Kenneth Heafield, Antonio Valerio Miceli Barone, and Philip Williams. 2017. The University of Edinburgh's Neural MT Systems for WMT17. CoRR, abs/1708.00726.

Rico Sennrich, Barry Haddow, and Alexandra Birch. 2016. Improving Neural Machine Translation Models with Monolingual Data. In Proceedings of the 54th Annual Meeting of the Association for Computational Linguistics (Volume 1: Long Papers), pages 86-96.

Jonathan Shen, Patrick Nguyen, Yonghui Wu, Zhifeng Chen, Mia X. Chen, Ye Jia, Anjuli Kannan, Tara N. Sainath, and Yuan Cao et al. 2019. Lingvo: a Modular and Scalable Framework for Sequence-toSequence Modeling. CoRR, abs/1902.08295.

Brian Thompson, Huda Khayrallah, Antonios Anastasopoulos, Arya D. McCarthy, Kevin Duh, Rebecca Marvin, Paul McNamee, Jeremy Gwinnup, Tim Anderson, and Philipp Koehn. 2018. Freezing Subnetworks to Analyze Domain Adaptation in Neural Machine Translation. In Proceedings of the Third Conference on Machine Translation: Research Papers, pages 124-132. Association for Computational Linguistics.
Nicola Ueffing, Gholamreza Haffari, and Anoop Sarkar. 2007. Semi-supervised model adaptation for statistical machine translation. Machine Translation, 21(2):77-94.

Ashish Vaswani, Noam Shazeer, Niki Parmar, Jakob Uszkoreit, Llion Jones, Aidan N Gomez, Łukasz Kaiser, and Illia Polosukhin. 2017. Attention Is All You Need. In Advances in Neural Information Processing Systems, pages 5998-6008.

Gholamreza Haffari Vu Cong Duy Hoang, Philipp Koehn and Trevor Cohn. 2018. Iterative Backtranslation for Neural Machine Translation. In Proceedings of the 2nd Workshop on Neural Machine Translation and Generation, volume 1, pages 18-24.

Marlies van der Wees, Arianna Bisazza, and Christof Monz. 2017. Dynamic Data Selection for Neural Machine Transaltion. In Proceedings of the 2017 Conference on Empirical Methods in Natural Language Processing, pages 1400-1410.

Yingce Xia, Tao Qin, Wei Chen, Jiang Bian, Nenghai Yu, and Tie-Yan Liu. 2017. Dual Supervised Learning. In International Conference on Machine Learning (ICML).

Hayahide Yamagishi, Shin Kanouchi, Takayuki Sato, and Mamoru Komachi. 2016. Controlling the voice of a sentence in Japanese-to-English neural machine translation. In Proceedings of the 3rd Workshop on Asian Translation (WAT2016), pages 203-210. 